DPSU-96-2

LMU-TPW 96-3

January, 1996

\title{
Soft Scalar Masses in String Models with Anomalous $U(1)$ symmetry
}

\author{
Yoshiharu Kawamura ${ }^{a}$ 用 and Tatsuo Kobayashi ${ }^{b}$ 田 \\ ${ }^{a}$ Department of Physics, Shinshu University \\ Matsumoto, 390 Japan \\ and \\ ${ }^{b}$ Sektion Physik, Universität München \\ Theresienstr. 37, D-80333 München, Germany
}

\begin{abstract}
We obtain the low-energy effective theory from string models with anomalous $U(1)$ symmetry. The feature of soft supersymmetry breaking scalar masses and some phenomenological implications are discussed. We show that it is, in general, difficult to keep the degeneracy and the positivity of squared soft scalar masses at the Planck scale.
\end{abstract}

\footnotetext{
*e-mail: ykawamu@gipac.shinshu-u.ac.jp

†Alexander von Humboldt Fellow

e-mail: kobayash@lswes8.ls-wess.physik.uni-muenchen.de
} 


\section{Introduction}

Superstring theories are powerful candidates for the unification theory of all forces including gravity. There are various approaches to explore 4dimensional (4D) string models, for example, the compactification on CalabiYau manifolds [1], the construction of orbifold models [2, 3] and so on. The effective supergravity theories (SUGRAs) have been derived based on the above approaches [4. The structure of SUGRA [5] is constrained by considering field theoretical non-perturbative effects such as a gaugino condensation [6] and stringy symmetries such as duality [7] besides of perturbative results.

Though the origin of supersymmetry (SUSY) breaking is unknown, soft SUSY breaking terms have been derived under the assumption that the SUSY is broken by $F$-term condensations of the dilaton field $S$ and/or moduli fields $T$ [8]. Some phenomenologically interesting features are predicted from the structure of soft SUSY breaking terms which are parameterized by a few number of parameters, for example, only two parameters such as a goldstino angle $\theta$ and the gravitino mass $m_{3 / 2}$ in the case with the overall moduli and the vanishing vacuum energy [9]. Further cases with multimoduli fields are discussed in Refs. [10].

Most of 4D string models have anomalous $U(1)$ symmetries. Some interesting features are pointed out in those models. Fayet-Iliopoulos $D$-term 11 is induced at one-loop level for anomalous $U(1)$ symmetry 12. . . As a result, some scalar fields necessarily develop vacuum expectation values(VEVs) and some gauge symmetries can break down 14.

Such a symmetry breaking generates an intermediate scale $M_{I}$, which is defined as the magnitude of VEVs of scalar fields, below the Planck scale $M_{P l}$. Using the ration $M_{I} / M_{P l}$, higher dimensional couplings could explain hierarchical structures in particle physics like the fermion masses and their mixing angles. Recently much attention has been paid to such a study on the fermion mass matrices [15, 16]. In Refs. [15], $U(1)$ symmetries are used to generate realistic fermion mass matrices and some of them are anomalous, while stringy selection rules on nonrenormalizable couplings are used in Refs. [16]. Hence it is interesting to examine what features 4D string models with anomalous $U(1)$ symmetry can show at low energy or whether we can construct a realistic model.

\footnotetext{
* Some conditions for absence of anomalous $U(1)$ are discussed in ref. 13.
} 
In this paper, we derive the low-energy theory from $4 \mathrm{D}$ string models with the anomalous $U(1)$ symmetry. We discuss the feature of soft supersymmetry breaking scalar masses and some phenomenological implications. In particular, we study the degeneracy and the positivity of squared scalar masses. This subject has not been completely examined in the literatures [17, 18].

\section{Structure of soft scalar mass}

Let us explain our starting point and assumptions first. We assume that $4 \mathrm{D}$ string models are described as the effective SUGRA at the Planck scale $M_{P l}$. The gauge group is $G=G_{S M}^{\prime} \times U(1)_{A}$ where $G_{S M}^{\prime}$ is a group which contains the gauge group of the standard model, $S U(3)_{C} \times S U(2)_{L} \times U(1)_{Y}$ as a subgroup and $U(1)_{A}$ is anomalous. This anomaly is canceled by the Green-Schwarz mechanism[19]. The chiral multiplets are classified into two categories. One is a set of $G_{S M}^{\prime}$ singlet fields with large VEVs denoted as $\Phi^{i}$. It is assumed that the SUSY is broken by those $F$-term condensations. Some of them have non-zero $U(1)_{A}$ charges and induce to the $U(1)_{A}$ breaking. $S$ and $T$ belong to $\left\{\Phi^{i}\right\}$. Here we treat only the overall moduli field $T$, but not several moduli fields. The second one is a set of $G_{S M}^{\prime}$ non-singlet fields $\Phi^{\kappa}$. For simplicity, we treat all $\Phi^{\kappa}$ 's as light fields whose masses are small compared with $M_{I}$. We denote the above two types of multiplet as $\Phi^{I}$.

We study only a simple case with the following assumptions to avoid a complication.

1. The $U(1)_{A}$ breaking scale is much higher than that of $G_{S M}^{\prime}$. We introduce one chiral matter multiplet $X$ with a large $\mathrm{VEV}$ of order $M_{I}$ to break $U(1)_{A}$.

2. The VEV of $X$ is much smaller than those of $S$ and $T$, i.e.

$$
\langle X\rangle \ll\langle S\rangle,\langle T\rangle=O(M)
$$

where $M$ is the gravitational scale defined as $M \equiv M_{P l} / \sqrt{8 \pi}$. Hereafter we take $M=1$.

3. Effects of threshold corrections and a $S-T$ mixing are small and neglected. 
It is straightforward to apply our method to more complicated situations. We will comment on some of them later.

Our starting SUGRA is determined by the following three gradients, that is, the Kähler potential $K$, the superpotential $W$ and the gauge kinetic function $f_{\alpha}$. Orbifold models lead to the following Kähler potential $K$ : 4, 20]

$$
\begin{aligned}
K= & -\ln \left(S+S^{*}+\delta_{G S}^{A} V_{A}\right)-3 \ln \left(T+T^{*}\right) \\
& +\left(T+T^{*}\right)^{n_{X}}|X|^{2}+\left(T+T^{*}\right)^{n_{\kappa}}\left|\Phi^{\kappa}\right|^{2}+\cdots
\end{aligned}
$$

where $\delta_{G S}^{A}$ is a coefficient of the Green-Schwarz mechanism to cancel $U(1)_{A}$ anomaly and $V_{A}$ is a vector superfield of $U(1)_{A}$. The dilaton field $S$ transforms nontrivially as $S \rightarrow S-i \delta_{G S}^{A} \theta(x)$ under $U(1)_{A}$ with the transformation parameter $\theta(x)$. The coefficient $\delta_{G S}^{A}$ is given as

$$
\delta_{G S}^{A}=\frac{1}{96 \pi^{2}} \operatorname{Tr} Q_{A}
$$

We estimate as $\left|\delta_{G S}^{A} / q_{X}\right|=O\left(10^{-1}\right) \sim O\left(10^{-3}\right)$ by using explicit models. ${ }^{\dagger}$ Here $q_{X}$ is a $U(1)_{A}$ charge of $X$. And $n_{I}$ 's are modular weights of matter multiplets $\Phi_{I}$. The same Kähler potential is derived from Calabi-Yau models with the large $T$ limit. If the $\mathrm{VEV}$ of $X$ is comparable with one of $T$, we should replace the second and third terms in Eq. (2) as

$$
-3 \ln \left(T+T^{*}-|X|^{2}\right)
$$

for the untwisted sector and

$$
-\ln \left[\left(T+T^{*}\right)^{3}-\left(T+T^{*}\right)^{n_{X}+3}|X|^{2}\right]
$$

for the twisted sector. The superpotential $W$ has $U(1)_{A}$ invariance. We examine its consequence at low energy without specifying the form of $W$ in this paper. Note that the term dependent on only $X$ is forbidden by the $U(1)_{A}$ invariance. The total Kähler potential $G$ is defined as $G \equiv K+\ln |W|^{2}$. The gauge kinetic function $f_{\alpha}$ is given as $f_{\alpha}=S$. For simplicity, here we assume the Kac-Moody levels satisfy $k_{\alpha}=1$, because our results on soft terms are independent of a value of $k_{\alpha}$. The scalar potential is given as

$$
V=V^{(F)}+V^{(D)},
$$

\footnotetext{
$\dagger$ For example, see Refs. [14, 21].
} 


$$
\begin{aligned}
V^{(F)} \equiv & e^{G}\left(G^{I}\left(G^{-1}\right)_{I}^{J} G_{J}-3\right), \\
V^{(D)} \equiv & \frac{1}{S+S^{*}}\left(D^{A}\right)^{2}+\frac{1}{S+S^{*}}\left(D^{a}\right)^{2} \\
= & \frac{1}{S+S^{*}}\left(\frac{\delta_{G S}^{A}}{S+S^{*}}+q_{X} K_{X} X+q_{\kappa} K_{\kappa} \Phi^{\kappa}\right)^{2} \\
& +\frac{1}{S+S^{*}}\left(K_{\kappa}\left(T^{a} \Phi\right)^{\kappa}\right)^{2},
\end{aligned}
$$

where the indexes $I, J, \ldots$ run all scalar species, the index $a$ runs the generators of $G_{S M}^{\prime}$ gauge group and the $U(1)_{A}$ charge of matter multiplet $\Phi^{I}$ is denoted as $q_{I}$. Note that the Fayet-Iliopoulos $D$-term 11] appears in $V^{(D)}$ for $U(1)_{A}$ if we replace $S$ by its VEV. The $U(1)_{A}$ is broken by the condensations of $S$ and $X$. The $U(1)_{A}$ breaking scale is of order $\langle N\rangle$ where $N$ is a Nambu-Goldstone multiplet. The gravitino mass is obtained as $m_{3 / 2}^{2}=e^{G}$. We assume that $V^{(F)} \leq O\left(m_{3 / 2}^{2} M^{2}\right)$.

Next we explain the procedure to obtain the low-energy theory.

1. We write down the scalar potential $V$ by using the variations $\Delta \Phi^{i}=$ $\Phi^{i}-\left\langle\Phi^{i}\right\rangle$. We treat $\Phi^{i}$ 's as dynamical fields.

2. We identify the Nambu-Goldstone multiplet $N$ related to $U(1)_{A}$ breaking whose mass is the same order of that of $U(1)_{A}$ gauge boson by calculating the scalar masses.

3. Then we solve the stationary conditions of the potential for $\Phi^{i}$ while keeping the light fields arbitrary and integrate out the heavy field $N$ by inserting the solutions into the scalar potential. Simultaneously we take the flat limit, while fixing $m_{3 / 2}$ finite.

We can obtain the scalar potential $V^{e f f}$ of the effective theory by the straightforward calculation[22]. Here we write down the result in a modelindependent manner as follows,

$$
\begin{aligned}
V^{e f f} & =V_{0}+V_{S U S Y}^{e f f}+V_{\text {Soft }}^{e f f}, \\
V_{0} & =\left\langle e^{G}\left(G^{i}\left(G^{-1}\right)_{i}^{j} G_{j}-3\right)\right\rangle, \\
V_{S U S Y}^{e f f} & =\left|\frac{\partial \hat{W}_{e f f}}{\partial \Phi^{\kappa}}\right|^{2}+\frac{1}{2} g_{a}^{2}\left(K_{\kappa}\left(T^{a} \Phi\right)^{\kappa}\right)^{2},
\end{aligned}
$$




$$
\begin{aligned}
V_{\text {Soft }}^{e f f}= & A \hat{W}_{e f f}+B^{\kappa}(\Phi) \frac{\partial \hat{W}_{e f f}}{\partial \Phi^{\kappa}}+H . c . \\
& +\left(m^{2}\right)_{\kappa}^{\lambda} \Phi^{\kappa} \Phi_{\lambda}^{*}+C_{\kappa \lambda} \Phi^{\kappa} \Phi^{\lambda}+H . c .
\end{aligned}
$$

where $g_{a}$ 's are the gauge coupling constants of $G_{S M}^{\prime}$. Here we use the relation $\langle S\rangle=1 / g_{\alpha}^{2}$ and omit the terms whose magnitudes are less than $O\left(m_{3 / 2}^{4}\right)$. Note that there is no $D$-term contribution on the cosmological constant $V_{0}$. We do not write down the explicit forms for the effective superpotential $\hat{W}_{\text {eff }}$, parameter $A$, field-dependent functions $B^{\kappa}$ or $C_{\kappa \lambda}$ since it is irrelevant to the later discussions.f We are interested in only chirality-conserving scalar mass $\left(m^{2}\right)_{\kappa}^{\lambda}$ in this paper. The formula is given as

$$
\begin{aligned}
\left(m^{2}\right)_{\kappa}^{\lambda}= & \left(m_{3 / 2}^{2}+V_{0}\right)\left\langle K_{\kappa}^{\lambda}\right\rangle+\left\langle F^{i}\right\rangle\left\langle\left(K_{i \kappa}^{\mu}\left(K^{-1}\right)_{\mu}^{\nu} K_{\nu}^{j \lambda}-K_{i \kappa}^{j \lambda}\right)\right\rangle\left\langle F_{j}^{*}\right\rangle \\
& +q_{\kappa} g_{A}^{2}\left\langle D^{A}\right\rangle\left\langle K_{\kappa}^{\lambda}\right\rangle \\
\left\langle D^{A}\right\rangle= & 2 M_{A}^{-2}\left\langle F^{i}\right\rangle\left\langle F_{j}^{*}\right\rangle\left\langle\left(D^{A}\right)_{i}^{j}\right\rangle,
\end{aligned}
$$

where $M_{A}$ is the mass of $U(1)_{A}$ gauge boson and $g_{A}$ is a gauge coupling constant of $U(1)_{A}$. The last term in Eq. (13) is so-called $D$-term contribution to the scalar masses [23].

We can apply the above result (9) - (14) to the effective SUGRA defined by (2) - (8). For the analysis of soft SUSY breaking parameters, it is convenient to introduce the following parameterization

$$
\begin{aligned}
& \left\langle e^{G / 2}\left(K_{S}^{S}\right)^{-1 / 2} G^{S}\right\rangle=\sqrt{3} C m_{3 / 2} e^{i \alpha_{S}} \sin \theta \\
& \left\langle e^{G / 2}\left(K_{T}^{T}\right)^{-1 / 2}\left(G^{T}+\left(K_{T}^{T}\right)\left(K_{X}^{T}\right)^{-1} G^{X}\right)\right\rangle=\sqrt{3} C m_{3 / 2} e^{i \alpha_{T}} \cos \theta
\end{aligned}
$$

where $\left(K_{j}^{i}\right)$ is a reciprocal of $\left(K_{j}^{i}\right)^{-1}$. The vacuum energy $V_{0}$ is written as

$$
\begin{aligned}
V_{0} & =3\left(C^{2}-1\right) m_{3 / 2}^{2}+V_{0}(X), \\
V_{0}(X) & \equiv\left\langle e^{G}\left(\left[\left(K_{X}^{X}\right)^{-1}-\left(K_{T}^{T}\right)\left|K_{X}^{T}\right|^{-2}\right]\left|G^{X}\right|^{2}\right)\right\rangle .
\end{aligned}
$$

Since $C^{2}$ should be positive or zero, we have a constraint $V_{0}(X) \leq 3 m_{3 / 2}^{2}+V_{0}$ from Eq. (17). In the case with $V_{0}=0$, it becomes as

$$
\left[\left(K_{X}^{X}\right)^{-1}-\left(K_{T}^{T}\right)\left|K_{X}^{T}\right|^{-2}\right]\left|G^{X}\right|^{2} \leq 3
$$

\footnotetext{
$\ddagger$ Consult the reference 22 if necessary.
} 
It gives a constraint on the VEVs of $X$ and $T$. Further a larger value of $V_{0}(X)$ in the above region means $C \ll 1$. Such a limit as $C \rightarrow 0$ corresponds to the "moduli-dominated" breaking, that is, $F^{S} \ll 1$ and $F^{T}$ and $F^{X}$ contribute to the SUSY-breaking. Note that this situation does not agree with the case of the moduli-dominated breaking without the anomalous $U(1)$ breaking $\sin \theta \rightarrow 0$.

The stationary conditions lead to $\langle D\rangle=O\left(m_{3 / 2}^{2}\right)$, i.e. $\left\langle\delta_{G S}^{A} /\left(S+S^{*}\right)+\right.$ $\left.q_{X} K_{X} X\right\rangle=0$ up to such an order. When we expand the scalar potential by using the variations for $\Phi^{i}$, we can identify the variation of Nambu-Goldstone multiplet $N$ as

$$
\begin{aligned}
\Delta N= & \frac{1}{a}\left[\frac{\Delta S+\Delta S^{*}}{2\langle S\rangle}+\left(1+\frac{\left\langle K_{\xi \xi}\right\rangle\langle X\rangle^{2}}{\left\langle K_{\xi}\right\rangle}\right) \frac{\Delta X+\Delta X^{*}}{\langle X\rangle}\right. \\
& \left.+\frac{\left\langle K_{\xi T}\right\rangle}{\left\langle K_{\xi}\right\rangle}\left(\Delta T+\Delta T^{*}\right)\right],
\end{aligned}
$$

where $\xi=|X|^{2}$ and

$$
a^{2}=\frac{1}{4\langle S\rangle^{2}}+\frac{1}{\langle X\rangle^{2}}\left(1+\frac{\left\langle K_{\xi \xi}\right\rangle\langle X\rangle^{2}}{\left\langle K_{\xi}\right\rangle}\right)^{2}+\left(\frac{\left\langle K_{\xi T}\right\rangle}{\left\langle K_{\xi}\right\rangle}\right)^{2} .
$$

This field $\Delta N$ has a heavy mass of order $O\left(\left|q_{X} \delta_{G S}^{A}\right|^{1 / 2} M\right)$, and the other linear combinations of $S, T$ and $X$ have light masses. 3 The mass of $U(1)_{A}$ gauge boson $M_{A}$ is given as

$$
M_{A}^{2}=2 g_{A}^{2}\left(\delta_{G S}^{A}{ }^{2}\left\langle K_{S}^{S}\right\rangle+q_{X}^{2}\left\langle K_{X}^{X}\right\rangle|\langle X\rangle|^{2}\right) .
$$

We can find that $\left|\delta_{G S}^{A} / q_{X}\right| \ll 1$ corresponds to $\langle X\rangle \ll\langle S\rangle,\langle T\rangle$. In this limit, $\Delta N$ and $M_{A}$ are as follows,

$$
\Delta N=\Delta X+\Delta X^{*}, \quad M_{A}^{2}=2 g_{A}^{2} q_{X}^{2}\left\langle K_{X}^{X}\right\rangle|\langle X\rangle|^{2} .
$$

Further $V_{0}(X)$ is negligible in this limit. Thus the scalar mass is rewritten as

$$
\begin{aligned}
m_{\kappa}^{2} & =m_{3 / 2}^{2}+V_{0}+\left(m_{F}^{2}\right)_{\kappa}+\left(m_{D}^{2}\right)_{\kappa}, \\
V_{0} & \equiv 3\left(C^{2}-1\right) m_{3 / 2}^{2}, \\
\left(m_{F}^{2}\right)_{\kappa} & \equiv m_{3 / 2}^{2} C^{2} n_{\kappa} \cos ^{2} \theta, \\
\left(m_{D}^{2}\right)_{\kappa} & \equiv m_{3 / 2}^{2} \frac{q_{\kappa}}{q_{X}}\left(1-C^{2} n_{X} \cos ^{2} \theta+6 C^{2} \sin ^{2} \theta\right),
\end{aligned}
$$

\footnotetext{
$\S$ We can estimate the order of their masses as $O\left(m_{3 / 2}\right)$ [22].
} 
where $\left(m^{2}\right)_{\kappa}^{\lambda}=m_{\kappa}^{2}\left\langle K_{\kappa}^{\lambda}\right\rangle$. Note that our result is not reduced to that obtained from the theory with Fayet-Iliopoulos $D$-term, which is derived from the effective SUGRA by taking the flat limit first, even in the limit that $\left|\delta_{G S}^{A} / q_{X}\right| \ll 1$. This disagreement originates from the fact that we regard $S$ and $T$ as dynamical fields, that is, we use the stationary conditions $\partial V / \partial \Phi^{i}=0$ to calculate $D$-term condensation. This mass formula plays a crucial role in the following discussion.

\section{Phenomenological implications of soft scalar mass}

Now we shall discuss phenomenological implications of our results, especially the degeneracy and the positivity of the squared soft scalar masses. Hereafter we take $V_{0}=0$, i.e. $C^{2}=1$. First we give a general argument by using the mass formula

$$
m_{\kappa}^{2}=m_{3 / 2}^{2}\left[1+n_{\kappa} \cos ^{2} \theta+\frac{q_{\kappa}}{q_{X}}\left(7-n_{X} \cos ^{2} \theta-6 \cos ^{2} \theta\right)\right] .
$$

Note that the coefficient of $q_{\kappa} / q_{X}$ in Eq. (28) is sizable. That could lead to a strong non-universality of soft scalar masses. Tा

\subsection{Degeneracy of soft scalar masses}

We obtain the difference of the soft masses as

$$
\frac{\Delta m^{2}}{m_{3 / 2}^{2}}=\Delta n \cos ^{2} \theta+\frac{\Delta q}{q_{X}}\left(7-n_{X} \cos ^{2} \theta-6 \cos ^{2} \theta\right),
$$

by using Eq. (28). The experiments for the process of flavor changing neutral current (FCNC) require that $\Delta m^{2} / m_{3 / 2}^{2} \lesssim 10^{-2}$ for the first and the second families in the case with $m_{\tilde{q}}^{2} \sim O(1) \mathrm{TeV}[25]$. Hence we should derive $\Delta m^{2} / m_{3 / 2}^{2} \approx 0$ within the level of $O\left(10^{-2}\right)$. Hereafter $a \approx 0$ denotes such a meaning.

\footnotetext{
ฯ Recently much work is devoted to phenomenological implications of the nonuniversality 24 .
} 
If $\Delta q / q_{X} \approx 0$, we have $\Delta m^{2} / m_{3 / 2}^{2}=\Delta n \cos ^{2} \theta$. In this case the limit $\cos ^{2} \theta \rightarrow 0$ leads to $\Delta m \rightarrow 0$. It corresponds to the dilaton-dominated breaking, where soft masses are universal [26, 9]. Unless $\Delta q / q_{X} \approx 0$, we needs "fine-tuning" on the value of $\cos \theta$ as

$$
\cos ^{2} \theta \approx \frac{7}{6+n_{X}-q_{X} \Delta n / \Delta q} .
$$

This "fine-tuning" is possible only in the case wherell

$$
1+\frac{\Delta n}{\Delta q} q_{X} \leq n_{X}
$$

Let us study the implication of Eq. (30). In the case with $\Delta n=0$, Eq. (30) is reduced $\cos ^{2} \theta \approx 7 /\left(6+n_{X}\right)$. Such a value of $\cos \theta$ is possible if $n_{X} \geq 1$. Since such modular weights require at least two oscillators, they are not obtained naturally 28. If we take more natural value, e.g. $n_{X}=-1$, Eq. (31) is reduced to $\Delta n q_{X} / \Delta q \leq-2$.

If Eq. (30) is satisfied, the soft scalar mass is written as

$$
m_{\kappa}^{2}=m_{3 / 2}^{2}\left[1+\frac{7\left(n_{\kappa}-\Delta n q_{\kappa} / \Delta q\right)}{6+n_{X}-\Delta n q_{X} / \Delta q}\right]
$$

\subsection{Positivity of squared soft scalar masses}

The condition for the positivity of $m_{\kappa}^{2}$ is written as

$$
-n_{\kappa}+\frac{q_{\kappa}}{q_{X}}\left(n_{X}+6\right) \leq\left(1+7 \frac{q_{\kappa}}{q_{X}}\right) \cos ^{-2} \theta .
$$

If $1+7 q_{\kappa} / q_{X}$ is positive, we can find a solution $\cos \theta$ of the above constraint for any $n_{\kappa}, n_{X}, q_{\kappa}$ and $q_{X}$. On the other hand, if $1+7 q_{\kappa} / q_{X}$ is negative, it leads to the following constraint;

$$
1+n_{\kappa} \geq \frac{q_{\kappa}}{q_{X}}\left(n_{X}-1\right)
$$

because $\cos ^{-2} \theta \geq 1$.

"In Ref. [27, the relation between modular weights and $U(1)$ charges is discussed as $q_{X} \Delta n / \Delta q=n_{X}$. This relation does not satisfy Eq. (31). 
Let us consider two extreme examples for the SUSY-breaking. One is the case of dilaton-dominated breaking $(\cos \theta=0)$. In this case we have

$$
m_{\kappa}^{2}=m_{3 / 2}^{2}\left(1+7 \frac{q_{\kappa}}{q_{X}}\right)
$$

The positivity of $m_{\kappa}^{2}$ requires that $1+7 q_{\kappa} / q_{X} \geq 0$. The other is that of moduli-dominated breaking $\left(\cos ^{2} \theta=1\right)$. In this case we have

$$
m_{\kappa}^{2}=m_{3 / 2}^{2}\left[1+n_{\kappa}+\frac{q_{\kappa}}{q_{X}}\left(1-n_{X}\right)\right]
$$

For example in the case with $n_{\kappa}=n_{X}=-1$, the positivity is realized only if $q_{\kappa} / q_{X}$ is positive.

In both cases of Eqs. (35) and (36), the fields with $q_{\kappa} / q_{X}<0$ can easily have negative squared scalar mass of $O\left(m_{3 / 2}^{2}\right)$ at the Planck scale. That implies that several fields develop VEV's and they trigger symmetry breakings. We can show that there exist fields with $q_{\kappa} / q_{X}<0$ for each gauge group other than $U(1)_{A}$. The reason is as follows. Let us assume the gauge group is $U(1)_{A} \times \prod_{\ell} G_{\ell}$. The Green-Schwarz anomaly cancellation mechanism requires that $C_{G_{\ell}}=\delta_{G S}^{A} k_{\ell}$ for any $\ell$, where $C_{G_{\ell}}$ is a coefficient of $U(1)_{A} \times G_{\ell}^{2}$ anomaly and $k_{\ell}$ is a Kac-Moody level of $G_{\ell}$. Through the $U(1)_{A}$ breaking due to the Fayet-Iliopoulos $D$-term, the field $X$ develops its VEV. Here its charge should satisfy $q_{X} \operatorname{Tr} Q_{A}<0$ and $q_{X} C_{G_{\ell}}<0$. Each gauge group $G_{\ell}$ always has fields $\Phi^{\kappa}$ which correspond nontrivial representation on its group and whose $U(1)_{A}$ charges satisfy $q_{X} q_{\kappa}<0$ because of $q_{X} C_{G_{\ell}}<0$. The $D$ term contribution on soft terms is very sizable. That could naturally lead to $m_{\kappa}^{2}<0$ except a narrow region and cause $G_{\ell}$ breaking.

Next we show that the scalar mass can be a source to break $G_{S M}^{\prime}$ by using the explicit model[21]. The model we study is the $Z_{3}$ orbifold model with a shift vector $V$ and Wilson lines $a_{1}$ and $a_{3}$ such as

$$
\begin{aligned}
& V=\frac{1}{3}(1,1,1,1,2,0,0,0)(2,0,0,0,0,0,0,0), \\
& a_{1}=\frac{1}{3}(0,0,0,0,0,0,0,2)(0,0,1,1,0,0,0,0), \\
& a_{3}=\frac{1}{3}(1,1,1,2,1,1,1,0)(1,1,0,0,0,0,0,0) .
\end{aligned}
$$


This model has a gauge group as

$$
G=S U(3)_{c} \times S U(2)_{L} \times S U(2)_{R} \times U(1)^{7} \times S O(8)^{\prime} \times S U(2)^{\prime}
$$

and matter multiplets as

$$
\begin{aligned}
& \mathrm{U}-\text { sec. } \quad: \quad 3\left[(3,2,1)_{0}+(\overline{3}, 1,2)_{0}+(1,2,2)_{0}\right] \\
& +3\left[(8,2)_{6}^{\prime}+(1,1)_{-12}^{\prime}\right] \text {, } \\
& \text { T- sec. } \quad: \quad 9\left[(3,1,1)_{4}+(\overline{3}, 1,1)_{4}\right]+15\left[(1,2,1)_{4}+(1,1,2)_{4}\right] \\
& \left(N_{O S C}=0\right) \quad+3(1,2,2)_{4}+3\left[(1,2,1)(1,2)_{-2}^{\prime}+(1,1,2)(1,2)_{-2}^{\prime}\right] \\
& 24(1,2)_{-2}^{\prime}+60(1,1,1)_{4}+3(1,1,1)_{-8}, \\
& \mathrm{~T}-\sec .\left(N_{O S C}=-1 / 3\right): \quad 9(1,1,1)_{4},
\end{aligned}
$$

where the number of suffix denotes the anomalous $\mathrm{U}(1)$ charge and $N_{O S C}$ is the oscillator number. This model has $\operatorname{Tr} Q_{A}=864$. Let us call the singlet fields in the untwisted sector $u$. Such fields have no charge other than the anomalous $U(1)$ charge.

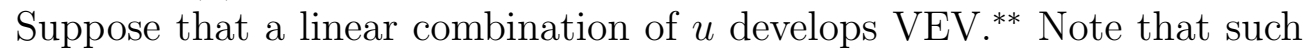
a VEV does not break any gauge groups except the anomalous $U(1)_{A}$. Our mass formula (28) holds in this case. In Table 1 , we give a ratio $m_{\kappa}^{2} / m_{3 / 2}^{2}$ for all species in two extreme cases, $\cos ^{2} \theta=0$ and $\cos ^{2} \theta=1$. More than half of the fields acquire negative squared masses and they could trigger a "larger" symmetry breaking including the dangerous color and/or charge symmetry breaking. In addition we have a strong non-universality of soft masses, i.e. $m_{\kappa}^{2}=O\left(10 m_{3 / 2}^{2}\right)$ for some fields while $m_{\kappa}^{2}=O\left(m_{3 / 2}^{2}\right)$ for others. However, in this model, soft masses are degenerate for squarks and sleptons with same quantum numbers under $G_{S M}$ because they have same quantum numbers under gauge group $G$ and same modular weights.

\section{Conclusions and discussions}

We study the effective field theory below the anomalous $U(1)$ symmetry breaking from the viewpoint of superstring theory. The $D$-term contributions on soft scalar masses are obtained and parameterized in terms of the

** This assumption should be justifed by solving the stationary conditions, but here we take it because non-pertabative effects in $W$ are not fully understood. 
goldstino angle. These contributions are very sizable. We find that the $F$ term contribution from the difference among modular weights and the $U(1)_{A}$ $D$-term contribution to scalar masses can destroy universality among scalar masses at $M$. This non-degeneracy endangers the discussion of the suppression of FCNC process. On the other hand, the difference among $U(1)$ charges is crucial for the generation of fermion mass hierarchy. It seems to be difficult to make two discussions compatible. As a byway, we can take a model that the fermion mass hierarchy is generated due to non-anomalous $U(1)$ symmetry and the SUSY is broken by the dilaton $F$-term condensation. For example, it is supposed that anomalies from the contributions of MSSM matters are cancelled out by those of extra matters in such a model. Moreover many fields acquire negative squared masses and they could trigger a "larger" symmetry breaking including the dangerous color and/or charge symmetry breaking. This type of symmetry breaking might be favorable in the case where $G_{S M}^{\prime}$ is a large group like a grand unified group. These results are very useful for model building.

There can exist other non-universal contributions to soft scalar masses in addition to $F$-term and $D$-term contributions discussed in this paper. We have not discussed them since we need a model-dependent analysis which is beyond the scope of this paper. Here we only give brief comments. On the breakdown of extra symmetries, there can exist $D$-term contributions, which give soft scalar masses a further non-universality. We can show that their contributions vanish for non-anomalous symmetries in the limit of dilaton dominant SUSY breaking. If there exist complex scalars whose masses are of order of the symmetry breaking scales, their F-term contributions can appear after integrating out them. Both of them should be considered at the symmetry breaking scales.

Further we have to take into account $T$-dependent threshold corrections and the $S$ - $T$ mixing at the one-loop level. Our approach is available in this case, too. Actually such corrections are rather small for most of cases.

The moduli fields have a problem in string cosmology because their masses are estimated as of $O\left(m_{3 / 2}\right)$ and they weakly couple with the observable matter fields, i.e. through the gravitational couplings [29]. They decay slowly to the observable matter fields. That makes the standard nucleosynthesis dangerous. In our model, some linear combinations of $S, T$ and $X$ remain light whose $F$-terms are of $O\left(m_{3 / 2} M\right)$ and break the SUSY. It is supposed that the couplings between such fields and observable fields are strongly 
suppressed to guarantee the stability of weak scale. Such a problem have to be considered for the light linear combinations, too.

\section{Acknowledgments}

The authors are grateful to J. Louis, H. Nakano, N. Polonsky, D. Suematsu and M. Yamaguchi for useful discussions. This work of Y.K. is supported by the Grant-in-Aid for Scientific Research $(\sharp 07740212)$ from the Japanese Ministry of Education, Science and Culture.

\section{References}

[1] P. Candelas, G. Horowitz, A. Strominger and E. Witten, Nucl. Phys. B258 (1985) 46.

[2] L. Dixon, J. Harvey, C. Vafa and E. Witten, Nucl. Phys. B261 (1985) 651; B274 (1986) 285.

[3] L.E. Ibáñez, J.E. Kim, H.P. Nilles and F. Quevedo, Phys. Lett. B191 (1987) 282;

Y. Katsuki, Y. Kawamura, T. Kobayashi, N. Ohtsubo, Y. Ono and K. Tanioka, Nucl. Phys. B341 (1990) 611.

[4] E. Witten, Phys. Lett. B155 (1985) 151;

S. Ferrara, C. Kounnas and M. Porrati, Phys. Lett. B181 (1986) 263.

[5] E. Cremmer, B. Julia, J. Scherk, S. Ferrara, L. Girardello and P. van Nieuwenhuizen, Phys. Lett. B79 (1978) 231; Nucl. Phys. B147 (1979) 105 ;

E. Cremmer, S. Ferrara, L. Girardello and A. van Proeyen, Phys. Lett. B116 (1982) 231; Nucl. Phys. B212 (1983) 413.

[6] J.P. Derendinger, L.E. Ibáñez and H.P. Nilles, Phys. Lett. B155 (1985) 65 ;

M. Dine, R. Rohm, N. Seiberg and E. Witten, Phys. Lett. B156 (1985) 65. 
[7] S. Ferrara, D. Lüst, A. Shapere and S. Theisen, Phys. Lett. B225 (1989) 363.

[8] L.E. Ibáñez and D. Lüst, Nucl. Phys. B382 (1992) 305;

B. de Carlos, J.A. Casas and C. Muñoz, Phys. Lett. B299 (1993) 234;

V.S. Kaplunovsky and J. Louis, Phys. Lett. B306 (1993) 269.

[9] A. Brignole, L.E. Ibáñez and C. Muñoz, Nucl. Phys. B422 (1994) 125.

[10] T. Kobayashi, D. Suematsu, K. Yamada and Y. Yamagishi, Phys. Lett. B348 (1995) 402;

A. Brignole, L.E. Ibáñez, C. Muñoz and C. Scheich, FTUAM 95/26, hep-ph/9508258.

[11] P. Fayet and J. Iliopoulos, Phys. Lett. B51 (1974) 416;

P. Fayet, Nucl. Phys. B90 (1975) 104.

[12] M. Dine, N. Seiberg and E. Witten, Nucl. Phys. B289 (1987) 589;

J. Atick, L. Dixon and A. Sen, Nucl. Phys. B292 (1987) 109;

M. Dine, I. Ichinose and N. Seiberg, Nucl. Phys. B293 (1987) 253.

[13] T. Kobayashi and H. Nakano, in preparation.

[14] A. Font, L.E. Ibáñez, H.P. Nilles and F. Quevedo, Nucl. Phys. B307 (1988) 109; Phys. Lett. B210 (1988) 101;

J.A. Casas, E.K. Katehou and C. Muñoz, Nucl. Phys. B317 (1989) 171;

J.A. Casas and C. Muñoz, Phys. Lett. B209 (1988) 214; B214 (1988) 63.

[15] M. Leurer, Y. Nir and N. Seiberg, Nucl. Phys. B398 (1993) 319; Nucl. Phys. B420 (1994) 468;

Y. Nir and N. Seiberg, Phys. Lett. B309 (1993) 337;

L.E. Ibáñez and G.G. Ross, Phys. Lett. B332 (1994) 100;

P. Binétruy and P. Ramond, Phys. Lett. B350 (1995) 49;

E. Dudas, S. Pokorski and C.A. Savoy, Phys. Lett. B356 (1995) 45.

[16] T. Kobayashi, Phys. Lett. B354 (1995) 264; Phys. Lett. B358 (1995) 253.

[17] H. Nakano, Preprint, KUNS 1257, HE(TH)94/05, hep-th/9404033. 
[18] The minimal SUGRA with Fayet-Iliopoulos D-term has been examined in A.H. Chamseddine and H. Dreiner, ETH-Preprint, ETH-TH/95-04, hep-ph/9504337.

[19] M.B. Green and J.H. Schwarz, Phys. Lett. B149 (1984) 117;

L.E. Ibáñez, Phys. Lett. B303 (1993) 55.

[20] M. Cvetič, J. Louis and B. Ovrut, Phys. Lett. B206 (1988) 227;

L.J. Dixon, V.S. Kaplunovsky and J. Louis, Nucl. Phys. B329 (1990) 27 ;

S. Ferrara, D. Lüst and S. Theisen, Phys. Lett. B233 (1989) 147.

[21] A. Font, L.E. Ibáñez, F. Quevedo and A. Sierra, Nucl. Phys. B331 (1990) 421.

[22] Y. Kawamura, Shinshu-Preprint, DPSU-95-7, hep-ph/9508290, to be published in Phys. Rev. D; DPSU-95-11, hep-ph/9511334, to be published in Prog. Theor. Phys. Supplement.

[23] M. Drees, Phys. Lett. B181 (1986) 279;

J.S. Hagelin and S. Kelley, Nucl. Phys. B342 (1990) 95;

A.E. Faraggi, J.S. Hagelin, S. Kelley and D.V. Nanopoulos, Phys. Rev. D45 (1992) 3272;

Y. Kawamura, H. Murayama and M. Yamaguchi, Phys. Rev. D51 (1995) 1337.

[24] A. Lleyda and C. Muñoz, Phys. Lett. B317 (1993) 82;

Y. Kawamura, H. Murayama and M. Yamaguchi, Phys. Lett. B324 (1994) 54;

T. Kobayashi, D. Suematsu and Y. Yamagishi, Phys. Lett. B329 (1994) 27 ;

N. Polonsky and A. Pomarol, Phys. Rev. Lett. 73 (1994) 2292;

D. Matalliotakis and H.P. Nilles, Nucl. Phys. B435 (1995) 115;

M. Olechowski and S. Pokorski, Phys. Lett. B344 (1995) 201;

Ph. Brax, U. Ellwanger and C.A. Savoy, Phys. Lett. B347(1995)269;

R. Altendorfer and T. Kobayashi, LMU-TPW-95-2, hep-ph/9503388, to be published in Int. J. Mod. Phys. A;

P.H. Chankowski, Z. Pluciennik, S. Pokorski and C.E. Vayonakis, Phys. Lett. B358(1995)264. 
[25] J. Ellis and D.V. Nanopoulos, Phys. Lett. B110 (1982) 44;

R. Barbieri and R. Gatto, Phys. Lett. B110 (1982) 211;

T. Inami and C.S. Lim, Nucl. Phys. B207 (1982) 533;

J. Hagelin, S. Kelly and T. Tanaka, Nucl. Phys. B415 (1994) 293.

[26] R. Barbieri, J. Louis and M. Moretti, Phys. Lett. B312 (1993) 451.

[27] E. Dudas, S. Pokorski and C.A. Savoy, Preprint, Saclay T95/094, MPIPTh 95-89, hep-ph/9509410.

[28] H. Kawabe, T. Kobayashi and N. Ohtsubo, Phys. Lett. B325 (1994) 77; Nucl. Phys. B434 (1995) 210;

T. Kobayashi, Int.J.Mod.Phys. A10 (1995) 1393.

[29] G.D. Coughlan, W. Fischler, E.W. Kolb, S. Raby and G.G. Ross, Phys. Lett. B131 (1983) 59;

B. de Carlos, J.A. Casas, F. Quvedo and E. Roulet, Phys. Lett. B318 (1993) 447;

T. Banks, D. Kaplan and A. Nelson, Phys.Rev. D49(1994)779;

J. Louis and Y. Nir, Nucl.Phys. B447(1995)18;

L. Randall and S. Thomas, Nucl.Phys. B449(1995)229. 
Table 1

\begin{tabular}{|c|c|c|c|c|c|}
\hline & Rep. & $\#$ & $q_{\kappa}$ & \multicolumn{2}{|c|}{$m_{\kappa}^{2} / m_{3 / 2}^{2}$} \\
& & & & $\cos ^{2} \theta=0$ & $\cos ^{2} \theta=1$ \\
\hline U-sec. & $Q_{L}(3,2,1)$ & 3 & 0 & 1 & 0 \\
& $Q_{R}(\overline{3}, 1,2)$ & 3 & 0 & 1 & 0 \\
& $H(1,2,2)$ & 3 & 0 & 1 & 0 \\
& $(8,2)^{\prime}$ & 3 & 6 & $-5 / 2$ & -1 \\
& $u(1,1)^{\prime}$ & 3 & -12 & 8 & 2 \\
\hline T-sec. & $(3,1,1)$ & 9 & 4 & $-4 / 3$ & $-5 / 3$ \\
$\left(N_{\text {OSC }}=0\right)$ & $(\overline{3}, 1,1)$ & 9 & 4 & $-4 / 3$ & $-5 / 3$ \\
& $L(1,2,1)$ & 15 & 4 & $-4 / 3$ & $-5 / 3$ \\
& $R(1,1,2)$ & 15 & 4 & $-4 / 3$ & $-5 / 3$ \\
& $(1,2,2)$ & 3 & 4 & $-4 / 3$ & $-5 / 3$ \\
& $(1,2,1)(1,2)^{\prime}$ & 3 & -2 & $13 / 6$ & $-2 / 3$ \\
& $(1,1,2)(1,2)^{\prime}$ & 3 & -2 & $13 / 6$ & $-2 / 3$ \\
& $(1,2)^{\prime}$ & 24 & -2 & $13 / 6$ & $-2 / 3$ \\
& $(1,1,1)$ & 60 & 4 & $-4 / 3$ & $-5 / 3$ \\
& $(1,1,1)$ & 3 & -8 & $17 / 3$ & $1 / 3$ \\
\hline T-sec. & $(1,1,1)$ & 9 & 4 & $-4 / 3$ & $-8 / 3$ \\
$\left(N_{O S C}=1 / 3\right)$ & & & & & \\
\hline
\end{tabular}

\section{Table Captions}

Table 1 The particle contents and the ratios of $m_{\kappa}^{2} / m_{3 / 2}^{2}$. In the third column, the degeneracy factors are shown. We refer to the chiral multiplets as $Q_{L}$ for left-handed quarks, $Q_{R}$ right-handed quarks, $H$ Higgs doublets, $L$ for left-handed leptons and $R$ for right-handed leptons. 